\title{
Induced pluripotent stem cells and severe combined immunodeficiency: merely disease modeling or potentially a novel cure?
}

Harald Mikkers ${ }^{1,2}$, Karin Pike-Overzet ${ }^{2}$ and Frank J.T. Staal ${ }^{2}$

For most, but not all, types of severe combined immunodeficiency (SCID) the underlying molecular defects are known, in principle allowing the cure of affected children via gene therapy. Typically such approaches have used autologous hematopoietic stem cells modified to express a therapeutic gene via $y$-retroviral vectors. Insertional mutagenesis has emerged as a significant risk for successful application of this type of gene therapy. Therefore, lentiviral vectors with a self-inactivating design have been developed. Recent advances in stem cell technology using induced pluripotent stem cells (iPSCs) allow an entire different approach to gene therapy for SCID and other genetic disorders, namely by correction of the affected gene in patient-specific iPSCs followed by hematopoietic differentiation. Here, we review these recent advances in the field from an efficacy and safety point of view.

\section{SEVERE COMBINED IMMUNODEFICIENCY}

Immunodeficiencies invariably refer to defects in the immune system that lead to an increased risk of infections. Severe combined immunodeficiency (SCID) is a heterogeneous disease characterized by lack of $\mathrm{T}$ lymphocytes and sometimes also $B$ and/or natural killer (NK) cells (1). Most infants develop opportunistic infections within the first 6 mo of life. The diagnosis is established by detecting lymphopenia, absence or very low numbers of $\mathrm{T}$ lymphocytes, and impaired T-cell proliferative responses to mitogens.

A number of genetic abnormalities can cause SCID. Worldwide, the most common form of SCID is X-linked SCID, caused by mutations in the gene coding for the Il2R $\gamma$ chain, resulting in SCID with a $\mathrm{T}^{-} \mathrm{B}^{+} \mathrm{NK}^{-}$phenotype, referring to the lack of T lymphocytes and NK cells, but the presence of B lymphocytes in these patients. The incidence is estimated to be roughly 1 in 65,000 live births (2) The lymphocytes of patients with X-linked SCID cannot respond to the several essential cytokines (interleukin (IL)-2, IL-4, IL-7, IL-9,
IL-15, and IL-21) needed for these cells to develop, survive, and fight infections. Although B cells are present, their function is severely impaired, not only because of a lack of T-cell help but also because of intrinsic B-cell defects.

Other forms of SCID are those with underlying deficiencies in the adenosine deaminase $(A D A)$ gene, recombinase-activating genes $(R A G)$, Artemis, or more rarely in the $C D 3$ genes, ZAP70 and IL7R (Figure 1). For many types of SCID, the underlying molecular defect is unknown. ADA-SCID patients fail to make T cells, B cells, and NK cells, experience recurrent infections, and fail to thrive (3). The ADA enzyme is found throughout the body but is most active in lymphocytes. ADA converts deoxyadenosine into nontoxic deoxyinosine. Mutations in the $A D A$ gene allow the buildup of deoxyadenosine to levels that are toxic to lymphocytes, in particular immature thymocytes.

The second or third most common form of SCID (depending on the genetic background of the population) is RAG-negative SCID. These patients have mutations in RAG1 or RAG2, which are required for the assembly of the T-cell receptor and B-cell receptor (4-6). As a consequence, RAG-SCID patients lack $\mathrm{B}$ and $\mathrm{T}$ cells and develop many serious, life-threatening infections, especially pneumonia, meningitis, and sepsis, as neonates.

Replacing the affected bone marrow with allogeneic healthy (stem) cells is currently the only established therapy for SCID. However, this treatment is complicated by adverse immune reactions of the donor cells, a slow immune reconstitution, and a lack of suitable donors for most patients. An alternative to allogeneic stem cells is genetically modified autologous stem cells in which the genetic defect is functionally corrected (i.e., by gene therapy). It should be noted, however, that also for SCID, haploidentical stem cell transplantation has become a valuable option for patients lacking a human leukocyte antigen-identical donor (7). These issues have mainly been worked out in children with leukemia, for whom suitable donors can often be found, using one of the parents, if necessary. However, delayed immune recovery and the risk of graft-vs.-host disease remain substantial problems that warrant gene repair via gene therapy with autologous cells as an alternative. 
a

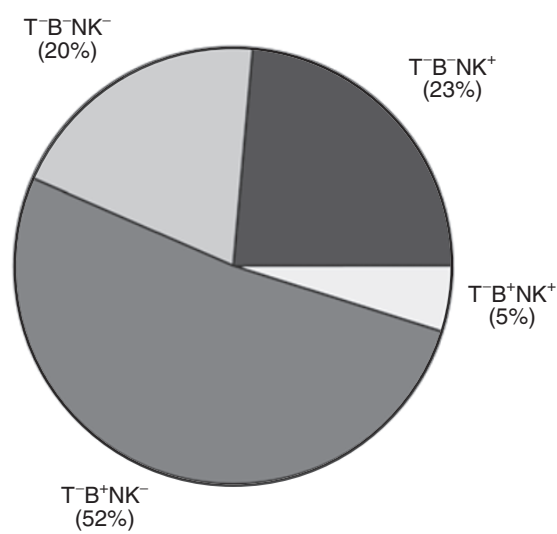

b

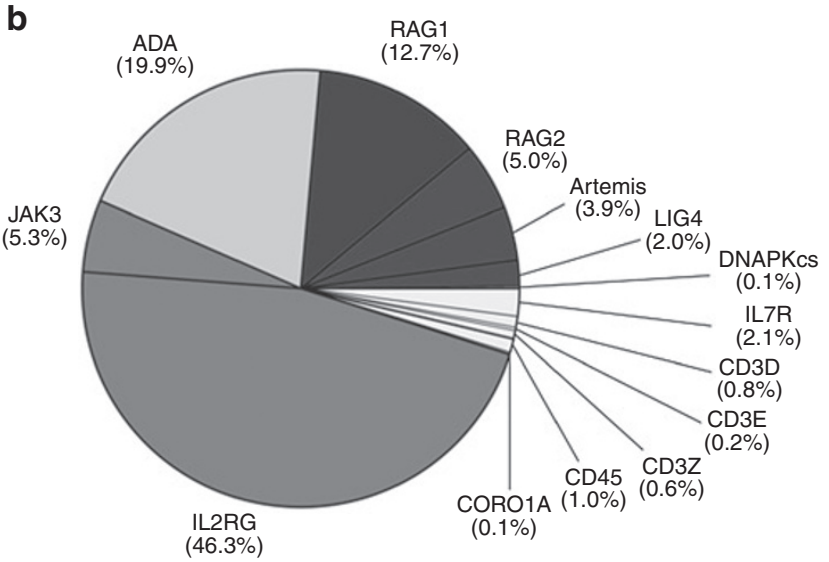

Figure 1. Mutation frequency in genes involved in severe combined immunodeficiency (SCID). Relative distribution of mutations in the genes known to be affected with SCID. (a) Genes are grouped according to the resulting absence and presence of $B$ and natural killer (NK) cells. (b) The mutation frequencies of individual genes are plotted. Data were obtained from http://bioinf.uta.fi/IDbases/, http://www.esid.org/, and known cases in the literature.

\section{GENE THERAPY FOR SCID}

Seminal work by Fischer and Cavazzana-Calvo (8) in Paris as well as Thrasher and Gaspar (9) in London for X-linked SCID and Bordignon and Aiuti (10-12) in Milan for ADASCID, has shown the clinical efficacy of gene therapy for various types of SCID using gene-corrected autologous stem cells. This work is covered by a number of excellent reviews (13-18), but will be briefly discussed here because of its relevance for other approaches of gene therapy using novel types of stem cells.

The two gene therapy trials for X-linked SCID have shown the clinical feasibility of introducing a therapeutic gene into hematopoietic stem cells (HSCs) $(8,9)$. Both X-linked SCID trials have been highly successful in many ways, showing long-lasting restoration of immunity. Immunodeficiency was restored and lymphocyte development was no longer blocked. However, the development of leukemia has appeared as a severe adverse effect in both trials (19). In all five reported cases ( $n=4$ of 10 children in the Paris trial and $n=1$ of 10 in the London trial), T-cell acute lymphoblastic leukemia occurred as a direct consequence of insertional mutagenesis by the retroviral vector used to deliver the therapeutic gene (20-24). Such severe adverse effects have not been reported in any of the Italian ADA-SCID patients; however, in a German trial using similar technology for Wiskott-Aldrich syndrome, one similar serious adverse event resulting from insertional mutagenesis has been reported (25).

In all of these cases of leukemia development, the strong viral promoter/enhancer sequences in the long terminal repeat of the vector that was used to deliver the therapeutic gene activated a cellular oncogene upon insertion in the host genome (Figure 2). In most instances, the insertion affected the LMO2 gene, a known T-cell acute lymphoblastic leukemia oncogene, which can block human thymopoiesis in an apparent preleukemic stage $(26,27)$. Recent work by Thrasher and coworkers demonstrated that the insertion near LMO2 is the first step in a multistep leukemogenesis program, involving somatic mutations in NOTCH1 and other leukemic events such as loss of the Arf tumor suppressor gene, collectively leading to full-blown leukemia (19).

It is hoped that the development of novel vectors, especially those in which the viral promoter/enhancer sequences have been rendered inactive (self-inactivating vectors), will significantly reduce the incidence of insertional mutagenesis. This will likely promote the safety and thus further clinical development of cells, which are genetically modified. Most investigators in the field have moved to such vectors, either in a $\gamma$-retroviral or lentiviral (HIV) backbone. For instance, self-inactivating lentiviral vectors have been developed in preclinical models for ADA-SCID (28), RAG-SCID (29,30), Artemis-SCID $(31,32)$, and agammaglobulinemias (33).

Another new development is the use of zinc-finger nucleases (34-36) or transcription activator-like effector nucleases (TALENS; ref. 37) to introduce specific genetic modifications in host genomes by homologous recombination (Figure 3). Gene correction of the endogenous genetic loci through homologous recombination overcomes the problems of insertional mutagenesis. However, in adult stem cells such as HSCs, this has proven to be technically challenging because the efficiency is low and HSCs cannot be efficiently expanded. In contrast, human and murine embryonic stem cells (ESCs) have in vitro unlimited self-renewal capacity, which would enable efficient genetic correction in a dish. Although adults do not possess pluripotent stem cells, recent exciting developments might have created novel opportunities for the treatment of SCID (38).

\section{PLURIPOTENT STEM CELLS}

Pluripotency is characterized by the ability to give rise to any cell type of our body. The best-characterized pluripotent stem cells are ESCs, which theoretically can self-renew indefinitely. The only way to isolate these cells is by culturing blastocysts and isolating the inner cell mass. The derivation of human ESC lines is, however, hindered by ethical concerns. Stem cell researchers have long been interested in generating pluripotent stem cells in an alternative way (39). In particular, a method to derive pluripotent cells from somatic cells would create tremendous opportunities. Such a 
a
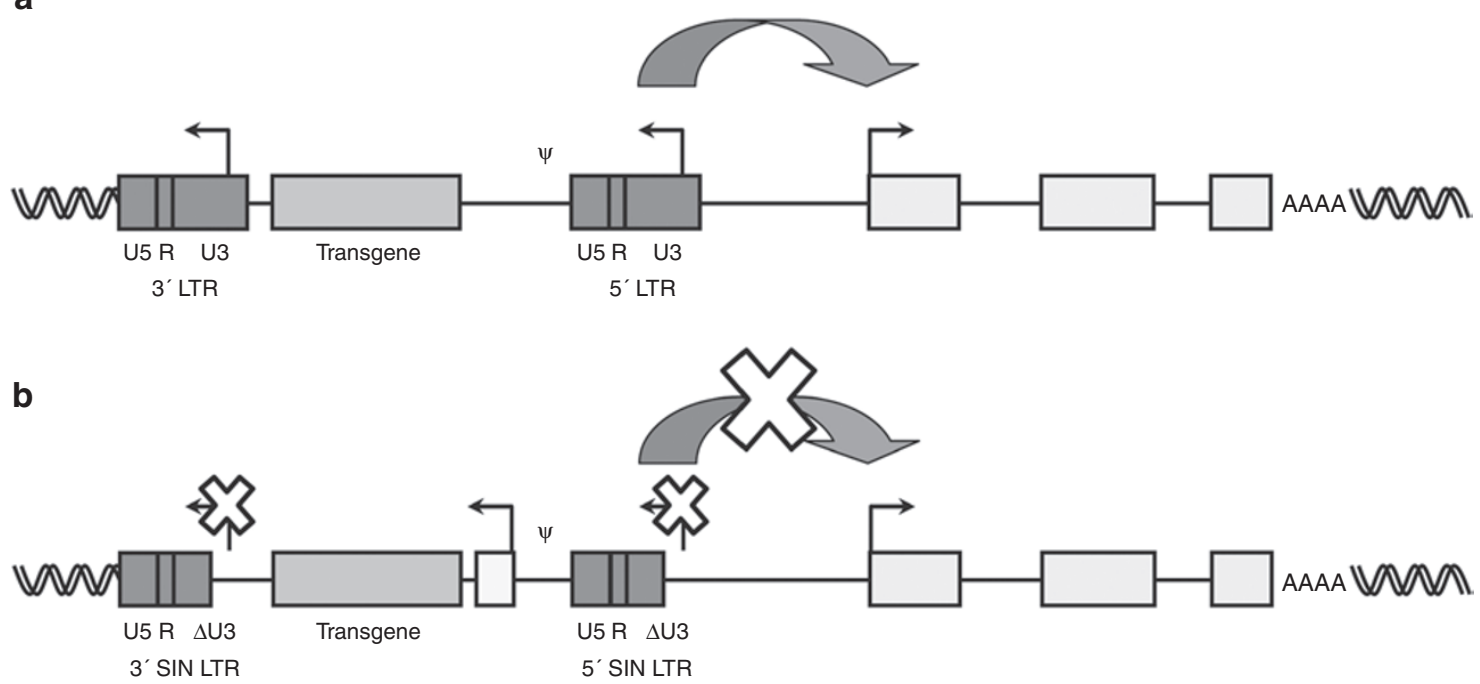

Figure 2. Insertional mutagenesis of integrating retroviral vectors. (a) An example of enhancer mutation as it may occur with a $\gamma$-retroviral or lentiviral vector integrated into the host genome. When integrated near a cellular gene, the enhancer element in the viral long terminal repeat (LTR) can upregulate this gene. U3, unique $3^{\prime}$ regulatory sequences; $\mathrm{R}$, repeat sequence; $\mathrm{U} 5$, unique $5^{\prime}$ regulatory sequences; $\Psi$, packaging signal. (b) In the self-inactivation $(\mathrm{SIN})$ versions of $\gamma$-retroviral or lentiviral vectors, viral enhancer and promoter sequences are deleted from the U3 region ( $\Delta U 3)$, eliminating the potential for activation of cellular genes by the viral LTRs. In this setting, an internal promoter is needed to drive the transgene expression.

method would not only bypass the ethical issues associated with ESCs but also allow the isolation of pluripotent stem cells from individuals with a specific genetic background. In addition, somatic cell-derived pluripotent cells create unique possibilities for cell replacement strategies because pluripotent stem cell banks that represent most haplotypes provide an inexhaustible source of any cell type. Provided that functional and safe HSCs can be generated from the banked pluripotent stem cells, congenital blood disorders that are currently only cured by bone marrow transplantations could be treated through this alternative source.

\section{INDUCED PLURIPOTENT STEM CELLS}

In 2006, Takahashi and Yamanaka used a simple but luminous approach to induce pluripotent stem cells from mouse tailtip fibroblasts (40). Exploiting a candidate gene-based screen, they identified a tetrad of transcription factors-Oct4, Sox2, $\mathrm{Klf} 4$, and $\mathrm{cMyc}-$ which are sufficient to revert a fibroblast into a state mirroring pluripotency. Although these first so-called induced pluripotent stem cells (iPSCs) did not fully behave like mouse ESCs, it was a crucial step forward to the immediate generation of pluripotent stem cells from somatic tissues. Soon after, cells that resembled mouse ESCs in all their facets were generated from tail-tip fibroblasts (41). These cells are pluripotent in vitro and in vivo. They generate teratomas containing derivatives of all three germ layers and contribute to all tissues during mouse development in chimeric mice, and iPSC-derived germline cells give rise to new offspring. Even the most stringent pluripotency test available for mouse cells (i.e., the ability of one single mouse iPSC to create a complete, viable, and fertile mouse upon injection into a blastocyst of tetraploid cells (tetraploid complementation)) was successfully passed by a number of iPSC lines (42-44). iPSCs are currently routinely being produced from somatic cells from different species, including humans, and from several tissues (45-47). Although the gene cocktail that induces the pluripotent state may differ, the resulting iPSC lines are similar to the existing ESC lines with respect to behavior, epigenome, transcription profiles, and proteome.

\section{SAFETY OF IPSCS}

Many patient-specific iPSC lines have been created and their number is rapidly increasing (48). These lines can be used to model diseases in a dish as well as in vivo. However, the first generation of iPSCs was made using conventional retroviral and lentiviral vector technology. These vector systems insert the genome of the host cells, which may act as insertional mutagens by altering endogenous gene expression, similar to the adverse effects observed in gene therapy trials.

Reprogramming into a pluripotent state, however, is dependent on the complete silencing of the pluripotency genes. Because most of the pluripotency genes have been associated with tumorigenesis, incomplete silencing or reactivation of the inserted proviruses can cause tumors (49-52). As a consequence, iPSCs used for in vivo disease modeling should preferentially be generated by different methods, and when future therapeutic applications are considered, nonintegrating delivery systems are required. Safe vector systems alone are not sufficient. It is largely enigmatic whether the whole reprogramming and culturing procedure affects the (epi)genome of iPSCs. Genetic drift has been reported in iPSC lines as copy number variations altered during iPSC generation and culture $(53,54)$. Whether the copy number variation changes constitute harmless natural selection occurring in any stressed and fast-growing cell population or represents a potentially dangerous selection process requires further investigation. Karyotyping has 
a

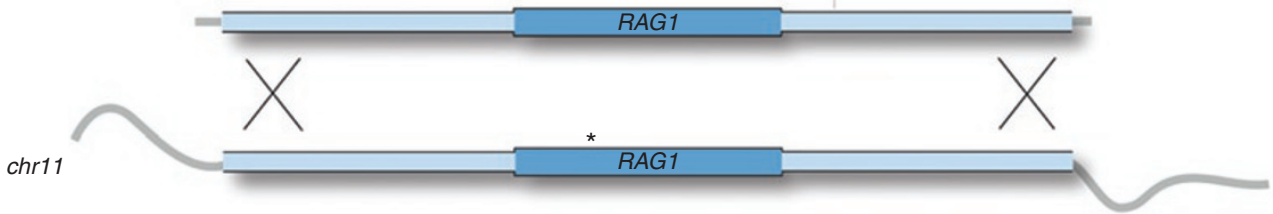

b

Gene repair by zinc-finger and TAL effector recombinases-mediated homologous recombination

ZFNs

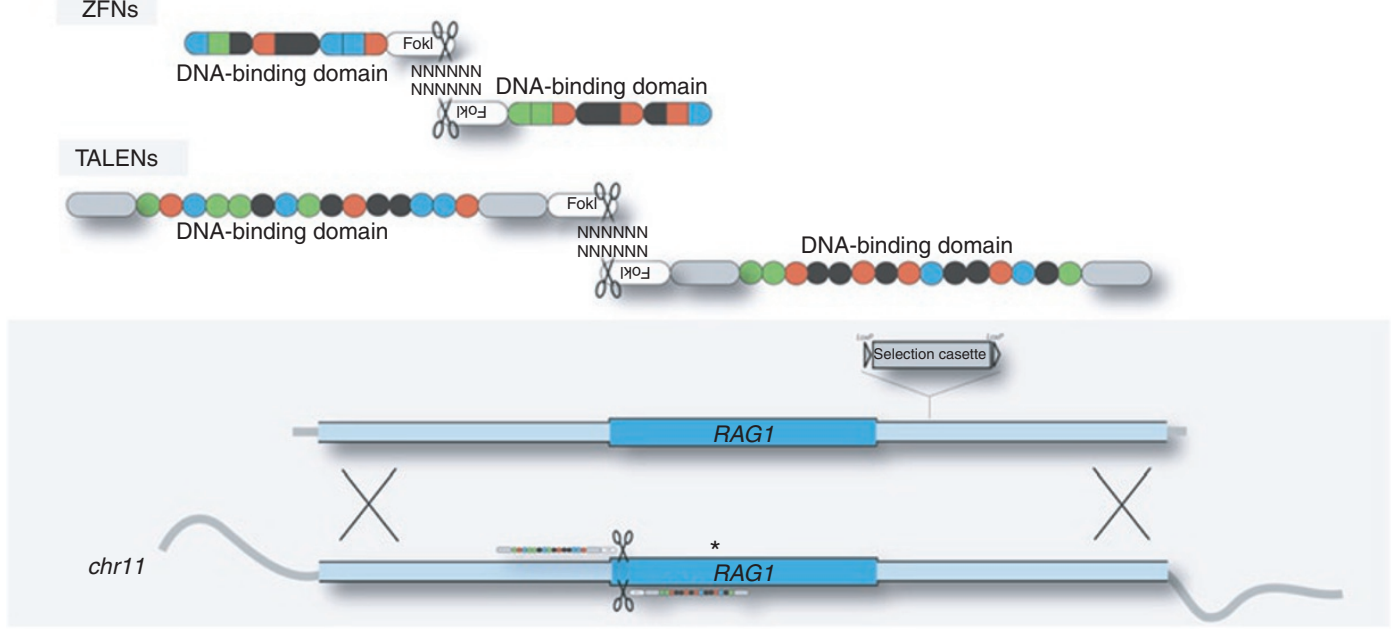

Figure 3. Repair of endogenous genes through homologous recombination. (a) Gene repair by homologous recombination. Conventional homologous recombination repair approaches are based on bringing a linearized targeting vector, in which a selection cassette is flanked by genomic sequences that are homologous to the locus/gene to be repaired, into the mutant cells. Proper homologous recombination events are rare in this setting, and positive selection of the cells using antibiotic selection markers is required to identify cells that have been correctly repaired. The strategy to repair one of the RAG1 mutant alleles (chromosome 11) in the case of RAG1-SCID is depicted. (b) Gene repair by zincfinger and transcription activator-like (TAL) effector recombinases-mediated homologous recombination. Engineered nucleases that bind and cleave the genomic DNA at specific places enhance the frequency of the homologous recombination. Two of these nucleases are zinc-finger nucleases (ZFNs) and TAL effector nucleases (TALENs). Both engineered proteins contain the Fokl nuclease domain, causing double-strand breaks, linked to a DNA-binding domain. DNA specificity is generated by either 3 zinc-fingers recognizing 9 nucleotides (ZFNs) or 15 TAL effector-derived repeat variable di-residues recognizing 15 nucleotides ( $\mathrm{A}$, green; T, red; $\mathrm{G}$, black; $\mathrm{C}$, blue). A TALEN strategy to repair one of the $R A G 1$ mutant alleles (chromosome 11) is depicted.

also revealed abnormalities in late-passage iPSC lines (55). In addition, single-point mutations potentially yielding a selective advantage have been observed in iPSC lines (56). These data may be caused by imperfect culture conditions or reprogramming stress but underscore the need for better characterization of iPSCs, their generation, and culture. Beside genomic issues, iPSCs may evoke an immune response upon transplantation of iPSCs as shown in a study using a syngeneic teratoma model (57). In conclusion, there are many safety issues concerning the generation and use of iPSCs that must be addressed before they can be utilized in transplantation settings.

\section{THERAPEUTIC POTENTIAL OF IPSCS}

Despite the uncertainties surrounding future iPSC use for cell replacement therapies, a proof-of-principle study using mouse iPSCs has clearly demonstrated the potential of iPSCs for therapeutic cell replacement purposes. In this study, iPSCs were derived from humanized sickle cell mice, in which the mouse $a$-globin genes were replaced with the human sickle

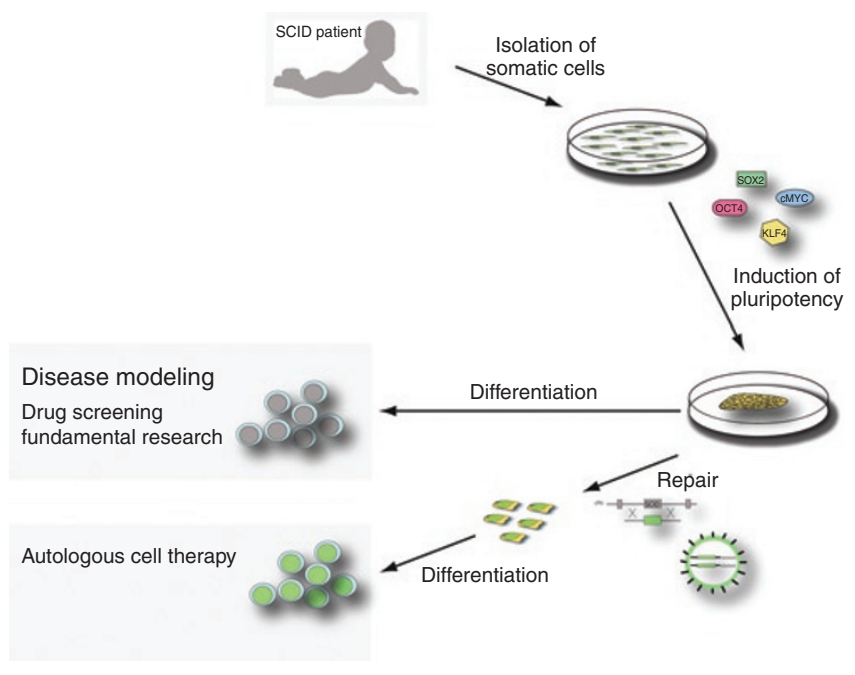

Figure 4. Use of SCID-specific induced pluripotent stem cells (iPSCs). Schematic representation of the use of iPSCs for severe combined immunodeficiency (SCID) diagnosis, drug screening, disease modeling, and therapy. 
Table 1. Potential use of and obstacles for iPSCs in PIDs

\begin{tabular}{ll}
\hline Potential use of iPSCs for PIDs & Current obstacles for efficient usage \\
\hline Disease modeling & $\begin{array}{l}\text { Robust lymphocyte and HSC } \\
\text { differentiation protocols do not yet exist }\end{array}$ \\
Drug screening & $\begin{array}{l}\text { Human iPSC-derived HSC-like cells fail to } \\
\text { repopulate in xenograft models }\end{array}$ \\
$\begin{array}{l}\text { Therapy: iPSC-derived HSCs } \\
\text { (iPSC banking) }\end{array}$ & $\begin{array}{l}\text { Availability of clinical material } \\
\text { Therapy: transplantation of } \\
\text { gene-repaired iPSCs }\end{array}$ \\
\hline
\end{tabular}

HSC, hematopoietic stem cell; iPSC, induced pluripotent stem cell; PID, primary immune deficiency.

globin variants $(58,59)$. The defective gene in the autologous iPSCs was repaired through homologous recombination, and HSCs were generated from the genetically corrected iPSCs. Transplantation of the ex vivo-generated corrected HSCs rescued the sickle cell phenotype. For a full in vivo functionality of the HSCs, the iPSCs required the expression of HoxB4. This brings forward the largest bottleneck concerning pluripotent stem cell-based hematopoietic studies because the evidence for the generation of pluripotent stem cell-derived HSCs that are functional in xenotransplantation protocols is scarce. Contribution to the mouse blood is in most cases low $(<1 \%)$ and of relatively short duration (60-65). Furthermore, the functionality of the generated lymphoid populations has never been demonstrated.

Nevertheless, one could potentially envision a similar strategy for the treatment of SCID: iPSCs generated from fibroblasts would allow gene correction of the affected locus via homologous recombination (38). Subsequently, selected iPSC clones could be differentiated into HSC-like cells and, after rigorous safety and efficacy testing, used in transplantation (Figure 4). Although many ethical and technical hurdles must be overcome in this procedure, as have reviewed previously, the underlying principles are attractive.

\section{iPSCS IN DISEASE MODELING FOR SCID}

A more modest goal in using iPSCs is their use for disease modeling. Disease- and patient-specific pluripotent cells can be used to model disease (66). Good disease models have been rare but are essential for research on disease pathogenesis, drug testing, and drug discovery. For the diagnosis of increasingly more and more immunodeficiencies, bone marrow punctures are no longer required and, therefore, are not undertaken. This leads to a shortage of transplantable stem cells to be used in in vitro and in vivo disease models (e.g., in nonobese diabetic-SCID or other mouse models). Here, iPSC technology may come in handy because iPSCs can be generated from peripheral blood cells or fibroblasts and then used in model systems. For some types of SCID, a small-molecule activator of the defective signaling pathways (e.g., defective signaling in JAK3-SCID) could be of therapeutic benefit. In such cases, iPSC-based disease modeling could provide a useful tool for drug screening (Table 1).

\section{CONCLUDING REMARKS}

The high efficacy of gene therapy for SCID compared with transplantation of non-human leukocyte antigen-identical HSCs (in which graft-vs.-host disease remains a major problem) provides a clear rationale for gene therapy (20). This therapy is currently performed using novel self-inactivating vectors. In addition, new approaches with iPSCs are currently under investigation by various laboratories. As the technology in this rapidly expanding field becomes more advanced and safer, the use of autologous stem cells, via iPSCs containing genes repaired by homologous recombination (38), is already providing valuable insight into the pathogenesis of blood-borne diseases and may, when proven safe, provide a novel treatment modality.

\section{ACKNOWLEDGMENTS}

We thank the members of the Staal laboratory for useful discussions.

\section{STATEMENT OF FINANCIAL SUPPORT}

The authors are supported by grants from the Netherlands Organization for Health Research and Development ZonMW (Innovational Research Incentives Scheme Veni grant 91610083), Kika (Children Cancer Free, 09/036), and the Landsteiner Foundation for Blood Transfusion Research (LSBR 0911).

\section{REFERENCES}

1. Fischer A. Human primary immunodeficiency diseases: a perspective. Nat Immunol 2004;5:23-30.

2. Nicholas S, Krance RA, Hanson IC, et al. Early versus delayed diagnosis of SCID: triumph versus tragedy. Clin Immunol 2011;139:360-2.

3. Gaspar HB, Aiuti A, Porta F, Candotti F, Hershfield MS, Notarangelo LD. How I treat ADA deficiency. Blood 2009;114:3524-32.

4. de Villartay JP. V(D)J recombination deficiencies. Adv Exp Med Biol 2009;650:46-58.

5. de Villartay JP, Lim A, Al-Mousa H, et al. A novel immunodeficiency associated with hypomorphic RAG1 mutations and CMV infection. J Clin Invest 2005;115:3291-9.

6. Niehues T, Perez-Becker R, Schuetz C. More than just SCID-the phenotypic range of combined immunodeficiencies associated with mutations in the recombinase activating genes (RAG) 1 and 2. Clin Immunol 2010;135:183-92.

7. Buckley RH, Schiff SE, Schiff RI, et al. Hematopoietic stem-cell transplantation for the treatment of severe combined immunodeficiency. $\mathrm{N}$ Engl J Med 1999;340:508-16.

8. Cavazzana-Calvo M, Hacein-Bey S, de Saint Basile G, et al. Gene therapy of human severe combined immunodeficiency (SCID)-X1 disease. Science 2000;288:669-72.

9. Gaspar HB, Parsley KL, Howe S, et al. Gene therapy of X-linked severe combined immunodeficiency by use of a pseudotyped gammaretroviral vector. Lancet 2004;364:2181-7.

10. Aiuti A, Cattaneo F, Galimberti S, et al. Gene therapy for immunodeficiency due to adenosine deaminase deficiency. N Engl J Med 2009;360:447-58.

11. Aiuti A, Vai S, Mortellaro A, et al. Immune reconstitution in ADA-SCID after PBL gene therapy and discontinuation of enzyme replacement. Nat Med 2002;8:423-5.

12. Bordignon C, Mavilio F, Ferrari G, et al. Transfer of the ADA gene into bone marrow cells and peripheral blood lymphocytes for the treatment of patients affected by ADA-deficient SCID. Hum Gene Ther 1993;4:513-20.

13. Fischer A, Hacein-Bey-Abina S, Cavazzana-Calvo M. 20 years of gene therapy for SCID. Nat Immunol 2010;11:457-60.

14. Aiuti A. Advances in gene therapy for ADA-deficient SCID. Curr Opin Mol Ther 2002;4:515-22.

15. Hacein-Bey S, Gross F, Nusbaum P, Yvon E, Fischer A, Cavazzana-Calvo $\mathrm{M}$. [Gene therapy of X-linked severe combined immunologic deficiency (SCID-X1)]. Pathol Biol 2001;49:57-66.

16. Kohn DB, Sadelain M, Glorioso JC. Occurrence of leukaemia following gene therapy of X-linked SCID. Nat Rev Cancer 2003;3:477-88. 
17. Pike-Overzet K, van der Burg M, Wagemaker G, van Dongen JJ, Staal FJ. New insights and unresolved issues regarding insertional mutagenesis in X-linked SCID gene therapy. Mol Ther 2007;15:1910-6.

18. Staal FJ, Pike-Overzet K, Ng YY, van Dongen JJ. Sola dosis facit venenum. Leukemia in gene therapy trials: a question of vectors, inserts and dosage? Leukemia 2008;22:1849-52.

19. Howe SJ, Mansour MR, Schwarzwaelder K, et al. Insertional mutagenesis combined with acquired somatic mutations causes leukemogenesis following gene therapy of SCID-X1 patients. J Clin Invest 2008;118:3143-50.

20. Cavazzana-Calvo M, Lagresle C, Hacein-Bey-Abina S, Fischer A. Gene therapy for severe combined immunodeficiency. Annu Rev Med 2005;56:585-602.

21. Fischer A, Abina SH, Thrasher A, von Kalle C, Cavazzana-Calvo M. LMO2 and gene therapy for severe combined immunodeficiency. N Engl J Med 2004;350:2526-7; author reply 2526-7.

22. Hacein-Bey-Abina S, von Kalle C, Schmidt M, et al. A serious adverse event after successful gene therapy for X-linked severe combined immunodeficiency. N Engl J Med 2003;348:255-6.

23. Hacein-Bey-Abina S, Von Kalle C, Schmidt M, et al. LMO2-associated clonal $\mathrm{T}$ cell proliferation in two patients after gene therapy for SCID-X1. Science 2003;302:415-9.

24. Thrasher AJ, Gaspar HB, Baum C, et al. Gene therapy: X-SCID transgene leukaemogenicity. Nature 2006;443:E5-6; discussion E6-7.

25. Persons DA, Baum C. Solving the problem of $\gamma$-retroviral vectors containing long terminal repeats. Mol Ther 2011;19:229-31.

26. Pike-Overzet K, de Ridder D, Weerkamp F, et al. Gene therapy: is IL2RG oncogenic in T-cell development? Nature 2006;443:E5; discussion E6-7.

27. Pike-Overzet K, de Ridder D, Weerkamp F, et al. Ectopic retroviral expression of LMO2, but not IL2Rgamma, blocks human T-cell development from CD34+ cells: implications for leukemogenesis in gene therapy. Leukemia 2007;21:754-63.

28. Mortellaro A, Hernandez RJ, Guerrini MM, et al. Ex vivo gene therapy with lentiviral vectors rescues adenosine deaminase (ADA)-deficient mice and corrects their immune and metabolic defects. Blood 2006;108:2979-88.

29. Lagresle-Peyrou C, Benjelloun F, Hue C, et al. Restoration of human B-cell differentiation into NOD-SCID mice engrafted with gene-corrected CD34+ cells isolated from Artemis or RAG1-deficient patients. Mol Ther 2008; 16:396-403.

30. Pike-Overzet K, Rodijk M, Ng YY, et al. Correction of murine Rag1 deficiency by self-inactivating lentiviral vector-mediated gene transfer. Leukemia 2011;25:1471-83.

31. Benjelloun F, Garrigue A, Demerens-de Chappedelaine C, et al. Stable and functional lymphoid reconstitution in artemis-deficient mice following lentiviral artemis gene transfer into hematopoietic stem cells. Mol Ther 2008;16:1490-9.

32. Mostoslavsky G, Fabian AJ, Rooney S, Alt FW, Mulligan RC. Complete correction of murine Artemis immunodeficiency by lentiviral vector-mediated gene transfer. Proc Natl Acad Sci USA 2006;103:16406-11.

33. Ng YY, Baert MR, Pike-Overzet K, et al. Correction of B-cell development in Btk-deficient mice using lentiviral vectors with codon-optimized human BTK. Leukemia 2010;24:1617-30.

34. Collin J, Lako M. Concise review: putting a finger on stem cell biology: zinc finger nuclease-driven targeted genetic editing in human pluripotent stem cells. Stem Cells 2011;29:1021-33.

35. Porteus $\mathrm{MH}$, Carroll D. Gene targeting using zinc finger nucleases. Nat Biotechnol 2005;23:967-73.

36. Urnov FD, Rebar EJ, Holmes MC, Zhang HS, Gregory PD. Genome editing with engineered zinc finger nucleases. Nat Rev Genet 2010;11:636-46.

37. Wood AJ, Lo TW, Zeitler B, et al. Targeted genome editing across species using ZFNs and TALENs. Science 2011;333:307.

38. Staal FJ, Baum C, Cowan C, et al. Stem cell self-renewal: lessons from bone marrow, gut and iPS toward clinical applications. Leukemia 2011;25:1095-102.

39. Stadtfeld M, Hochedlinger K. Induced pluripotency: history, mechanisms, and applications. Genes Dev 2010;24:2239-63.
40. Takahashi K, Yamanaka S. Induction of pluripotent stem cells from mouse embryonic and adult fibroblast cultures by defined factors. Cell 2006;126:663-76.

41. Takahashi K, Tanabe $\mathrm{K}$, Ohnuki $\mathrm{M}$, et al. Induction of pluripotent stem cells from adult human fibroblasts by defined factors. Cell 2007;131:861-72.

42. Zhao XY, Lv Z, Li W, Zeng F, Zhou Q. Production of mice using iPS cells and tetraploid complementation. Nat Protoc 2010;5:963-71.

43. Boland MJ, Hazen JL, Nazor KL, et al. Adult mice generated from induced pluripotent stem cells. Nature 2009;461:91-4.

44. Zhao XY, Li W, Lv Z, et al. iPS cells produce viable mice through tetraploid complementation. Nature 2009;461:86-90.

45. Pera MF, Tam PP. Extrinsic regulation of pluripotent stem cells. Nature 2010;465:713-20.

46. Staerk J, Dawlaty MM, Gao Q, et al. Reprogramming of human peripheral blood cells to induced pluripotent stem cells. Cell Stem Cell 2010;7:20-4.

47. Yu J, Vodyanik MA, Smuga-Otto K, et al. Induced pluripotent stem cell lines derived from human somatic cells. Science 2007;318:1917-20.

48. Yamanaka S. Patient-specific pluripotent stem cells become even more accessible. Cell Stem Cell 2010;7:1-2.

49. Vojnits K, Bremer S. Challenges of using pluripotent stem cells for safety assessments of substances. Toxicology 2010;270:10-7.

50. Miura K, Okada Y, Aoi T, et al. Variation in the safety of induced pluripotent stem cell lines. Nat Biotechnol 2009;27:743-5.

51. Hentze H, Soong PL, Wang ST, Phillips BW, Putti TC, Dunn NR. Teratoma formation by human embryonic stem cells: evaluation of essential parameters for future safety studies. Stem Cell Res 2009;2:198-210.

52. Duinsbergen D, Salvatori D, Eriksson M, Mikkers H. Tumors originating from induced pluripotent stem cells and methods for their prevention. Ann N Y Acad Sci 2009;1176:197-204.

53. Laurent LC, Ulitsky I, Slavin I, et al. Dynamic changes in the copy number of pluripotency and cell proliferation genes in human ESCs and iPSCs during reprogramming and time in culture. Cell Stem Cell 2011;8:106-18.

54. Hussein SM, Batada NN, Vuoristo S, et al. Copy number variation and selection during reprogramming to pluripotency. Nature 2011;471:58-62.

55. Chen G, Ye Z, Yu X, et al. Trophoblast differentiation defect in human embryonic stem cells lacking PIG-A and GPI-anchored cell-surface proteins. Cell Stem Cell 2008;2:345-55.

56. Gore A, Li Z, Fung HL, et al. Somatic coding mutations in human induced pluripotent stem cells. Nature 2011;471:63-7.

57. Zhao T, Zhang ZN, Rong Z, Xu Y. Immunogenicity of induced pluripotent stem cells. Nature 2011;474:212-5.

58. Zou J, Mali P, Huang X, Dowey SN, Cheng L. Site-specific gene correction of a point mutation in human iPS cells derived from an adult patient with sickle cell disease. Blood 2011;118:4599-608.

59. Hanna J, Wernig M, Markoulaki S, et al. Treatment of sickle cell anemia mouse model with iPS cells generated from autologous skin. Science 2007;318:1920-3.

60. Morishima T, Watanabe K, Niwa A, et al. Neutrophil differentiation from human-induced pluripotent stem cells. J Cell Physiol 2011;226:1283-91.

61. Senju S, Hirata S, Motomura Y, et al. Pluripotent stem cells as source of dendritic cells for immune therapy. Int J Hematol 2010;91:392-400.

62. Lei F, Haque R, Weiler L, Vrana KE, Song J. T lineage differentiation from induced pluripotent stem cells. Cell Immunol 2009;260:1-5.

63. Ye Z, Zhan H, Mali P, et al. Human-induced pluripotent stem cells from blood cells of healthy donors and patients with acquired blood disorders. Blood 2009;114:5473-80.

64. Lengerke C, Grauer M, Niebuhr NI, et al. Hematopoietic development from human induced pluripotent stem cells. Ann N Y Acad Sci 2009;1176:219-27.

65. Ledran MH, Krassowska A, Armstrong L, et al. Efficient hematopoietic differentiation of human embryonic stem cells on stromal cells derived from hematopoietic niches. Cell Stem Cell 2008;3:85-98.

66. Unternaehrer JJ, Daley GQ. Induced pluripotent stem cells for modelling human diseases. Philos Trans R Soc Lond, B, Biol Sci 2011;366:2274-85. 